\title{
Diagnostic Performance of SD Bioline, Accurate, Fortress and Encode Compared to the Mindray CLIA 1200i Test in Volunteer Blood Donors at Kinshasa University Hospital
}

\author{
Alain Vandersal Salaboni Mungezi ${ }^{1}$, Benjamin Longo-Mbenza ${ }^{2,3}$, Jacques Bikaula Ngwidiwo1, \\ Héritier Mawalala Malengele ${ }^{1}$, Charles Mbendi Nlombi², Antoine Tshimpi Wola Yaba', \\ Bienvenu Kuyangisa Boloko ${ }^{1}$, Aliocha Nkodila Natuhoyila ${ }^{3,4^{*}}$, Mireille Solange Nganga Nkanga ${ }^{1}$ \\ ${ }^{1}$ Department of Clinical Biology, University of Kinshasa, Kinshasa, Democratic Republic of the Congo \\ ${ }^{2}$ Department of Internal Medicine, University of Kinshasa, Kinshasa, Democratic Republic of the Congo \\ ${ }^{3}$ Department of Public Health, Lomo University Research, Kinshasa, Democratic Republic of the Congo \\ ${ }^{4}$ Department of Family Medicine and Primary Care, Protestant University of Congo, Congo, Democratic Republic of the Congo \\ Email: *nkodilaaliocha@gmail.com
}

How to cite this paper: Mungezi, A.V.S., Longo-Mbenza, B., Ngwidiwo, J.B., Malengele, H.M., Nlombi, C.M., Yaba, A.T.W., Boloko, B.K., Natuhoyila, A.N. and Nkanga, M.S.N. (2021) Diagnostic Performance of SD Bioline, Accurate, Fortress and Encode Compared to the Mindray CLIA 1200i Test in Volunteer Blood Donors at Kinshasa University Hospital. Open Journal of Medical Microbiology, 11, 308-322. https://doi.org/10.4236/ojmm.2021.114020

Received: September 6, 2021

Accepted: November 28, 2021

Published: December 1, 2021

Copyright $\odot 2021$ by author(s) and Scientific Research Publishing Inc. This work is licensed under the Creative Commons Attribution International License (CC BY 4.0).

http://creativecommons.org/licenses/by/4.0/ (c) (i) Open Access

\begin{abstract}
Background and Purpose: The hepatitis $\mathrm{C}$ prevalence rate is among the highest the world. Thus, in the context of transfusion safety, WHO has made it compulsory to screen blood bags by rapid diagnostic orientation tests (TROD) validated in regions that do not have the capacity to use more sophisticated technologies. The purpose of this study is to assess the performance of the TRODs commonly used in Kinshasa. Methods: This was a cross-sectional and analytical study carried out from August 26, 2019 to January 20, 2020 in 200 voluntary blood donors at the University Clinics of Kinshasa (CUK). The detection of anti-HCV antibodies was carried out by Mindray (gold standard) and the SD Bioline, Encode, Fortress and Accurate tests. The diagnostic performance of TRODs was evaluated compared to the Mindray test. Results: Of a total of 200 donors, $112(56.0 \%)$ were male versus 88 (44.0\%) female with a sex ratio of $1 \mathrm{M}$ : $1 \mathrm{~F}$. HCV seroprevalences by Mindray, and other tests were $29.5 \%, 28.0 \%, 27.0 \%, 27.0 \%$ and $31.5 \%$, respectively; the Se, Sp, VPP, VPN, SD Bioline, Accurate, Fortress and Encode were 74.6\%, 92.9\%, 81.5\%, 89.7\%, respectively; 74.6\%, 92.9\%, 81.5\%, 89.7\%; 74.6\%, 91.4\%, $78.6 \%, 89.6 \%$; 69.5\%, 70.0\%, 65.1\%, 84.4\%. Conclusion: The diagnostic performance of TRODs evaluated in this study does not meet European Union standards $(\mathrm{Se}=100 \%$; $\mathrm{Sp}>99.5 \%)$. The Ministry of Health should promote large-scale validation of TRODs based on World Health Organization guidelines.
\end{abstract}




\section{Keywords}

HCV, TROD, Diagnostic Performance, Screening, Seroprevalence

\section{Introduction}

The hepatitis $\mathrm{C}$ virus (HCV) is one of the major causes of morbidity (prevalence and incidence) and mortality in the world. The World Health Organization (WHO) reports an annual incidence of 3 to 4 million people with HCV [1]. However, its prevalence varies from one geographic region to another but also from one population to another. Thus, WHO recognizes HCV infection as a public health problem in the general population [2], and among voluntary blood donors (DBS) [3].

In the Democratic Republic of Congo (DRC), the prevalence of HCV in blood donors ranged from $2.3 \%$ to $13.24 \%$ in the HD population [3] [4]. Faced with this situation, screening for chronic HCV infection is now becoming a public health challenge, especially with the therapeutic revolution in hepatitis $\mathrm{C}$ and with the arrival in 2010 of new direct-acting antivirals (DAAs) [5].

The problem of HCV detection in rich countries, emerging countries and developing countries (DCs) with limited resources, considers the following concepts: HCV screening; rapid rather than rapid diagnosis of HCV; the HCV rapid diagnostic orientation test (TROD); acceptability of HCV detection; the feasibility of HCV TRODs and the decision tree (algorithm) for HCV diagnostic referral. In resource-limited countries, TRODs are offered for the detection of total anti-HCV antibodies which are generally detected by new ELISA methods including chemiluminescence immunoassay (CLIA), in view of their diagnostic performance. However, TRODs are limited in the detection of HCV compared to nucleic acid tests (NAT) and genotypic tests which allow the quantification of the HCV RNA and the detection of the different genotypes by the PCR considered as a test reference for diagnostic confirmation [6] [7].

Like the other tests already introduced in 2003 (SD Bioline, Fortress, Encode, Accurate) on screening actions, the University Clinics of Kinshasa (CUK) recently introduced in 2019 for the first time the chemiluminescence immunoassay methods which also have a high sensitivity and specificity than the classic ELISA and the RIA until now considered as the reference method [8] [9] [10].

At CUK, TRODs are used alternatively in the biological qualification of blood bags and/in the diagnosis of hepatitis. Unfortunately, the majority of TRODs used for the detection of anti-HCV Ab in the DRC have not been evaluated. And yet, the WHO recommends (thus overriding the recommendation of the WHO, that) to use in the diagnostic management of the HCV in second instance the quantification of the RNA of the virus and the detection of the viral genotypes circulating in serum or plasma.

Unlike the human immunodeficiency virus (HIV), there is no consensus or 
even no strategy on the use of TRODs for HCV. The existence of a large number of TROD VHC on the Congolese market, the multitude of health centers, both public and private, as well as those of blood transfusion structures, thus constitute a challenge of good governance for the Congolese authorities.

The problematic of this study underlined a large gap between the countries with planned health system and the DRC plagued by poverty, true nature of several challenges as motivation of the present study which sets as objective to evaluate the performances of the TROD currently used in Kinshasa.

\section{Methods}

\subsection{Design, Setting and Period of Study}

This was a cross-sectional and analytical study that was carried out at the Kinshasa University Hospital/University of Kinshasa during the period from August 26, 2019 to January 20, 2020.

\subsection{Study Population}

The study population consisted of all blood donors received at the University Clinics of Kinshasa. The sampling was non-probability and convenience. Taking into account the donors received, 200 were included in the study according to certain criteria. All voluntary (voluntary) blood donors of both sexes were included in the study; any volunteer donor who has not donated blood within 3 months and whose age was between 18 and 65 years old. Volunteer donors who donated within 3 months of the previous donation; pregnant for female donors; showing symptoms of a chronic and debilitating infection; those lacking information on sex and age as well as those who refused to participate in the study were excluded.

\subsection{Collection of Data}

A pre-established sheet made it possible to collect the sex and age (non-modifiable factors) of voluntary donors and the results of various serological analyzes (search for anti-HCV antibodies).

\subsection{Laboratory Analysis}

The quantitative research of anti-HCV antibodies was carried out using the Mindray CL1200i automatic device (BS/CUK, Nanshan, Shenzhen/China) which detects HCV qualitatively and quantitatively and which uses the principle of chemiluminescence immunoassay (CLIA), defined as the emission of electromagnetic radiation caused by a chemical reaction that produces light. The presence or absence of anti-HCV antibodies in the sample is determined by comparing the chemiluminescent signal in the reaction to the cut-off signal determined from the system calibration. A cutoff index (COI) is calculated as follows: sample RLU/cutoff RLU value.

Samples with a COI $<1.00$ are non-reactive in the Mindray Anti HCV Assay. 
Samples with a COI $\geq 1.00$ are initially reactive in the Mindray Anti HCV Assay.

TRODs (Bioline, Fortress, Encode and Accurate) were used for the qualitative research of anti HCV antibodies. All these TRODs follow the same principle: immunochromatography, designed for the detection of specific antibodies to $\mathrm{HCV}$, in human serum, plasma or whole blood.

The presence of a colored band in the test region $(\mathrm{T})$ indicates a positive result. A colored band will always appear in control region $\mathrm{C}$ to serve as an indicator of the validity of the procedure for the proper execution of the test and strip.

Mindray anti-HCV quality control (QC) was performed at least once every 24 hours after loading a new reagent cartridge or after each calibration. QCs are recommended to verify the reliability of the assay system (Negative and Positive). QC results should be within acceptable ranges. If a QC is outside the specified range, the associated test results are invalid and the samples must be retested. Quality control was also ensured for each TROD of interest, it being understood that the anti-HCV antibody tests helped to obtain the reliability, precision and accuracy of the results (validity and reproducibility) in the present study.

The quality control of the statistical analyzes and the results of the present study were also ensured.

Each TROD complied with the instructions respectively published by the manufacturer.

The qualities of the analytical methods were inherent in the CUK laboratory for the benchmark test. Thus, the conditions were met for the introduction (precision of the objectives), the review of the literature (reduction of information bias) and the Mindray assay methods.

Intrinsic Indicators of the Mindray Benchmark Test.

Only Mindray (chemiluminescenceimmuno assay) used in the city of Kinshasa province is considered as a reference test (Gold standard).

Evaluation and validation of the performance and accuracy of each TROD of interest.

The use of the immunochromatographic results of each TROD was based on the evaluation and validation previously carried out during quality control. To do so, the interview questionnaire was structured and standardized for each voluntary blood donor and the encoding of demographic variables and TRODs was carried out by entering data on the Lenovo (China) laptop computer.

Completeness and consistency tests were used for the systematic cleaning of the file (the database).

A Carré-Latin (Table 1) defined the HCV seropositivity (diagnostic screening-orientation according to its performance of the possible or probable disease, if not absence of the disease) of the 4 TRODs of interest compared to the seroprevalence of $\mathrm{HCV}$ according to the Mindray benchmark test (definite disease). 
Table 1. Comparison of the precision indicators of the different TRODs compared to the Mindray reference test.

\begin{tabular}{ccccc}
\hline & \multicolumn{4}{c}{ MINDRAY } \\
\cline { 2 - 5 } Test & $\begin{array}{c}\text { Simple } \\
\text { correlation } \\
\text { coefficient }\end{array}$ & L+ & $\begin{array}{c}\text { Coefficient } \\
\text { Symetricuncertainty }\end{array}$ & $\begin{array}{c}\text { Kappa } \\
\text { (Mesure of } \\
\text { agreement) }\end{array}$ \\
\hline FORTRESS & 0.671 & 88.2 & 0.368 & 0.671 \\
ACCURATE & 0.693 & 94.2 & 0.396 & 0.692 \\
SDBIOLINE & 0.693 & 94.2 & 0.396 & 0.692 \\
ENCODE & 0.529 & 54.5 & 0.222 & 0.528 \\
\hline
\end{tabular}

Thus, the HCV seroprevalence of each TROD $=\mathrm{A}+\mathrm{B} / \mathrm{A}+\mathrm{B}+\mathrm{C}+\mathrm{D}=$ total study population.

The HCV seroprevalence according to the Mindray reference test is equal to A + $\mathrm{C} / \mathrm{A}+\mathrm{B}+\mathrm{C}+\mathrm{D}=$ total study population.

\subsection{Operational Definitions}

Sociodemographic variables were defined by gender (Male vs. Female) and age, which were considered non-modifiable variables.

The seroprevalence of HCV was defined by the presence of anti-HCV antibodies according to Mindray, reference test and according to the TRODs.

\subsection{Statistical Analyzes}

The categorical variables (nominal and qualitative: sex, age group, HCV seroprevalence rate according to Mindray and the 4 TRODs, diagnostic performance indicator) were presented as a proportion (percentage).

The quantitative variable (continuous: anti-HCV antibody level according to Mindray) was presented by quartile in the form of a figure.

The HCV seroprevalence percentages according to Mindray were compared between two groups (male versus female) by the chi-square test.

The percentages between 3 age groups were compared using the chi-square trend over linear association by linear association: biological gradient or response effect dose.

Sensitivity (Se), specificity (Sp), negative predictive value (NPV) and positive predictive value (PPV) were used to assess the performance of the 4 TRODs compared to Mindray as Gold Standard with calculation of the Kappa index and the Youden index to determine the degree of performance of each test.

The $\mathrm{p}$ value $<0.05$ defined the threshold of statistical significance. All statistical analyzes were performed using SPSS software (Statistical Package for Social Sciences) version 23.1 (New York, USA).

\subsection{Ethical Considerations}

The data were collected anonymously and confidentially. The privacy and con- 
fidentiality of the respondents were safeguarded. The three fundamental principles of ethics were respected at the time of the study, namely: the principle of respect for the person, that of beneficence, and that of justice. The protocol for this research study was conducted in accordance with the Declaration of Helsinki.

\section{Results}

\subsection{Participant Response Rate}

Of the 240 volunteer blood donors, 200 donors participated from start to finish with a response rate of $83.0 \%$.

\subsection{Sex and Age of the Study Population}

In the present study, there were 112 male blood donors versus 88 female blood donors with a sex ratio of 1 Male: 1 Female (Figure 1).

The mean age of blood donors was $34.83 \pm 7.76$ years. The age group under 30 was more represented. Several age groups were noted according to different thresholds defining advancement in age/chronology. There were $72,60,48$, and 20 DBS respectively in age $<30$ years, age equal 30 - 39 years, age equal $40-49$ years and age $\geq 50$ years of the population of study (Figure 2).

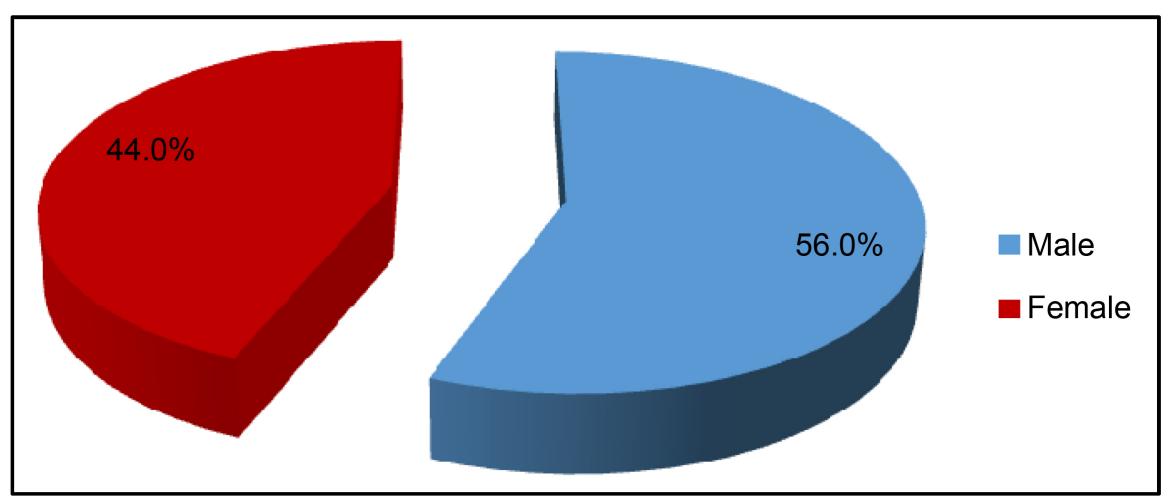

Figure 1. Distribution of the study population by sex.

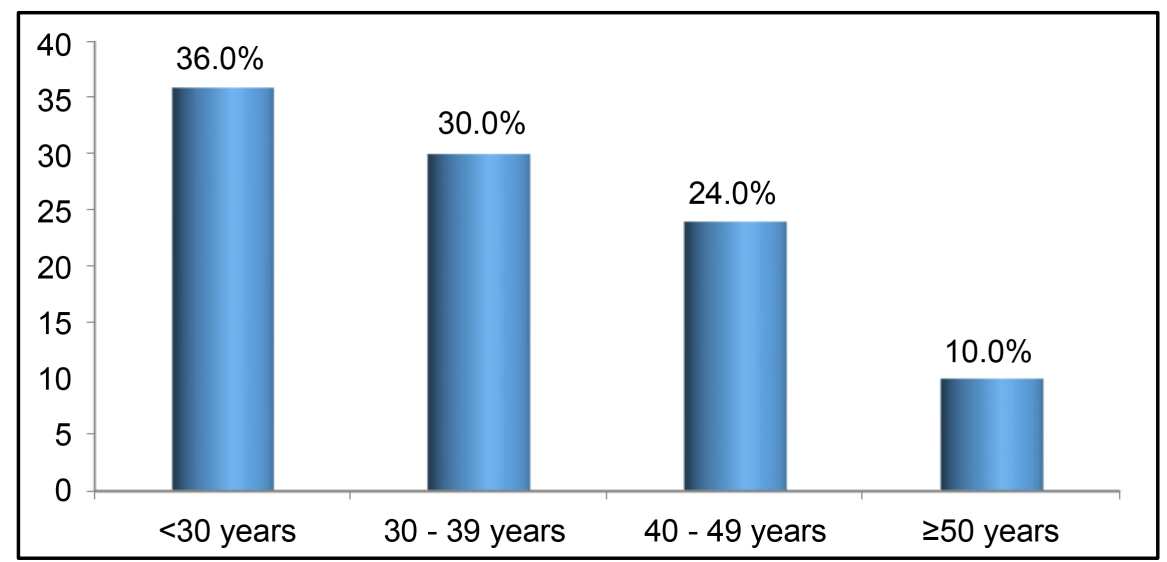

Figure 2. Distribution of the study population by age. 
The statistical characteristics of the distribution of S/CO ratio of anti HCV Abs according to the Mindray test in the study population are shown in Figure 3. With a mean of 3.3743; a standard error of the mean of 46,478 and a median of 80,000 .

Qualitative response of anti-HCV antibodies according to Mindray.

Of all the DBSs, 59 had hepatitis $\mathrm{C}$ according to the S/CO threshold ratio $\geq 1$ according to the Mindray reference test. Thus nearly $30 \%$ of the DBS were infected with HCV (Figure 4).

Qualitative response of anti-HCV antibodies according to TROD.

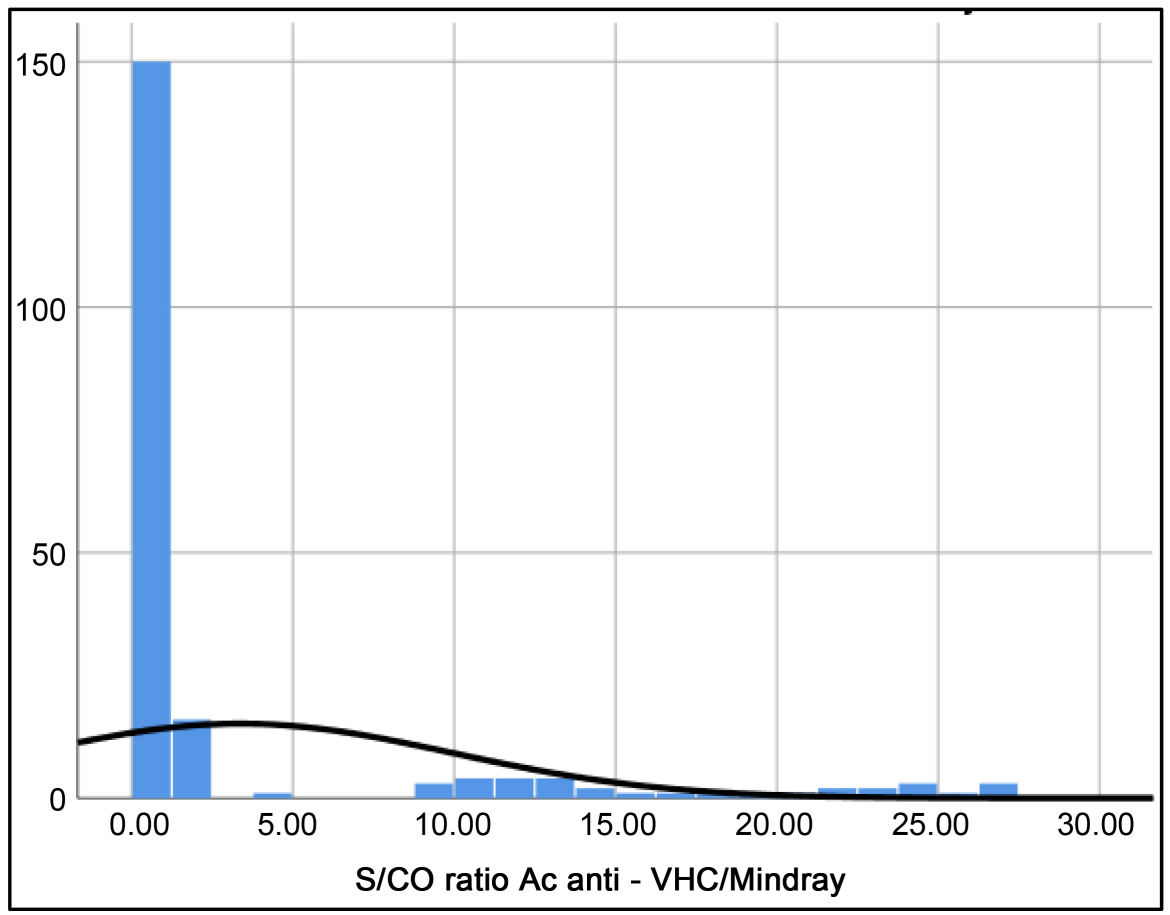

Figure 3. Asymmetric distribution with several modes.

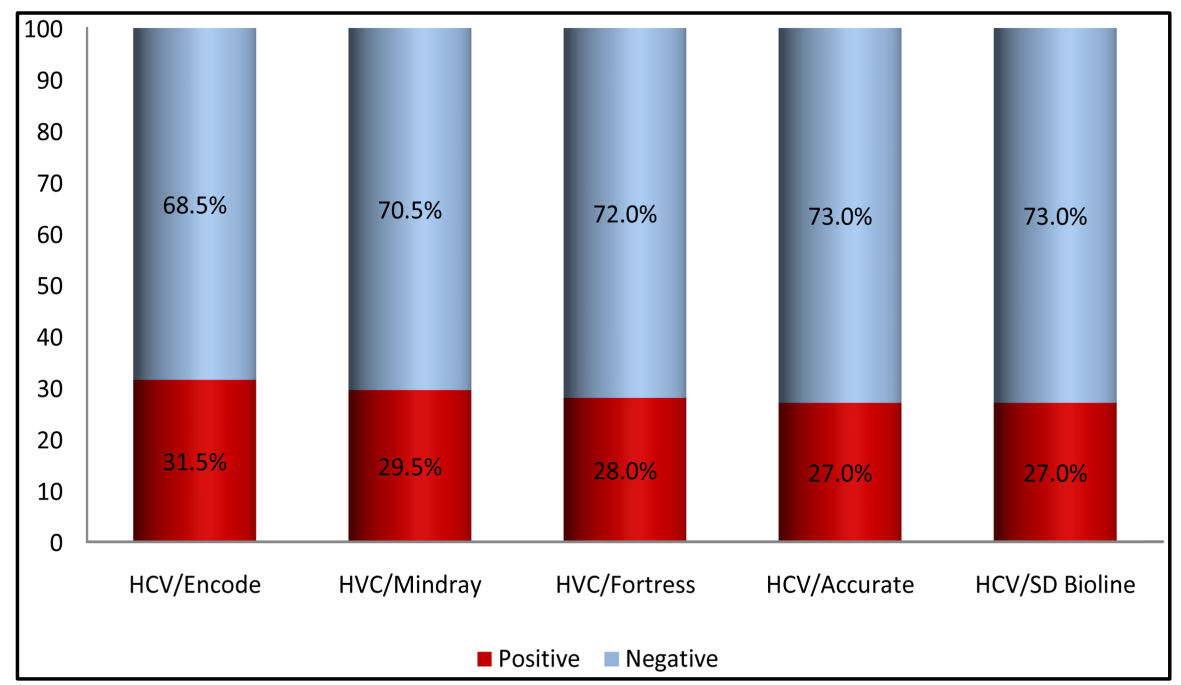

Figure 4. HCV seroprevalence according to the test used. 
Fortress test: Of all DBSs, 56 tested positive for HCV, thus less than a third of the study population had HCV infection. Accurate test Positive tests for HCV according to the accurate test were reported in 54 DBS among the entire study population: less than a third with hepatitis $\mathrm{C}$ screened. SD Bioline test: Among all DBSs, 54 tested positive for HCV according to the SD Bioline test: as with the Accurate test, less than a third of the population was screened for hepatitis $\mathrm{C}$. Encode test: Almost $32 \%$ of the total population $(n=63)$ tested positive for HCV according to the Encode test (Figure 4).

Diagnostic assessment of HCV seroprevalence by 4 TROD compared to the reference MINDRAY test.

Precision, reproducibility, repeatability of the anti-HCV antibody assay by Mindray.

The Mindray coefficient of variation of anti-HCV antibody assay was estimated at $1.9 \%$, reflecting the accuracy of a benchmark test.

Comparison of the precision indicators of the 4 TRODs of diagnostic interest compared to the Mindray reference test.

The diagnostic accuracy of the 4 TRODs of interest: Fortress, Accurate, SD Bioline and Encode is summarized in Table 1. With regard to these indicators, Accurate and SD Bioline's diagnostic accuracy was very good according to the Mindray benchmark test and Fortress's diagnostic accuracy was good according to the Mindray test. On the other hand, the diagnostic precision of the Encode test was quite good according to the Mindray reference test (Table 1).

Diagnostic performance indicators for the $4 \mathrm{HCV}$ TRODs according to the Mindray benchmark test.

The intrinsic and extrinsic indicators of diagnostic performance for each TROD of interest were compared to the Mindray reference test (Table 2).

Compared to the Mindray reference test, the SD Bioline test followed by the Accurate test and the Fortress test respectively exhibited good diagnostic performance for $\mathrm{HCV}$ in terms of sensitivity, specificity, positive and negative predictive values, while performance Encode's diagnostic compared to the Mindray reference test was low in terms of sensitivity, specificity, and positive predictive value. The table shows that the sensitivity of the 4 TRODs is still within the limit values, on the other hand a high specificity for FORTRESS, ACCURATE and

Table 2. Diagnostic performance indicators for 4 TRODs of interest in HCV according to Mindray.

\begin{tabular}{ccccc}
\hline Probability & FORTRESS & ACCURATE & SDBIOLINE & ENCODE \\
\hline Se (\%) & 74.6 & 74.6 & 74.6 & 69.5 \\
Sp (\%) & 91.4 & 92.9 & 92.9 & 70 \\
VPP (\%) & 78.6 & 81.5 & 81.5 & 65.1 \\
VPN (\%) & 89.6 & 89.7 & 89.7 & 84.4 \\
Indice de & 0.7 & 0.7 & 0.7 & 0.4 \\
Youden & & & & \\
\hline
\end{tabular}


SDBIOLINE but low for ENCODE. This indicates that the ENCODE is not to be used for the diagnosis of HCV.

\section{Discussion}

This chapter has commented on the overall results according to the objectives achieved. Indeed, this study examined the level of diagnostic performance of SD Bioline, Encode, Fortress and Accurate compared to the chemiluminescence immunoassay (reference test).

\subsection{Demographic Characteristics of DBS}

The seroprevalence according to the Mindray reference test was therefore considered to be the definite viral disease explained by demographic factors. On the other hand, the TROD SD Bioline, Accurate, Fortress and Encode provided respective hierarchical HCV seroprevalences in screening tests and rapid diagnostic orientation tests for the decision in the scientific management of HCV in CUKs. The present study estimated an epidemic-like HCV seroprevalence rate of $29.5 \%$ according to Mindray in DBS. This HCV seroprevalence rate of close to $30 \%$ according to chemiluminescence/Mindray was in the worldwide range of rates between $0.03 \%$ and $81.6 \%$ of HCV. Diagnostic assessment of HCV seroprevalence by 4 TROD compared to the Mindray reference test. Several studies in the literature [11] [12] [13] as well as the present study evaluated the performance of TRODs. Bivariate correlation between the 4 TRODs and the Mindray reference test to detect the prevalence of anti-HCV antibodies in DBS. This study underscored the high level of diagnostic accuracy of the Accurate and SD Bioline rapid tests with a close to 1 bivariate correlation with the Mindray benchmark test. However, the calculation of the Youden index, close to 1 [14], the best parameter of diagnostic precision, considered SD Bioline, Accurate and Fortress to be very good tests for the detection of HCV against the Encode test considered less precise to detect HCV in DBS. Indeed, the HCV prevalence rates of $28 \%, 27 \%$ and $27 \%$ respectively determined by Fortress, Accurate, and SD Bioline were comparable to the HCV prevalence rate of $29.5 \%$ determined by the Mindray reference test. Among the TRODs, except the Encode test with an overestimation of the prevalence of HCV of the order of $32 \%$ and poor diagnostic performance indicators $(\mathrm{Se}=69.5 \%$; $\mathrm{Sp}=70 \%$; $\mathrm{PPV}=65.1$; $\mathrm{NPV}=84.4$; Youden index $=0.4$; Kappa concordance $=0.524$ ); the rest of the TRODs including Fortress $(\mathrm{Se}=74.6 \%$; $\mathrm{Sp}=91.4 \%$; VPP $=78.6 \%$; NP $=89.6 \%$ Youden index $=0.7$; Kappa concordance $=0.671)$, Accurate $(\mathrm{Se}=74.6 \% ; \mathrm{Sp}=92.9 \%$; $\mathrm{VPP}=81.5 \% ; \mathrm{NPV}=89.7 \%$ Youden index $=0.7 ;$ Kappa concordance $=0.692)$, and SD Bioline ( $\mathrm{Se}=74.6 \%$; $\mathrm{Sp}=92.9 \%$; PPV $=81.5 \%$; NPV $=89.7 \%$ Youden's index $=0.7$; Kappa concordance $=0.692$ ) were considered to be very good screening tests and diagnostic guidance for HCV in DBS at CUK. Diagnostic performance indicator of SD Bioline, Accurate, Encode and Fortress against Mindraychemiluminescence immunoassay 
This interpretation of the results for the purpose of diagnostic guidance is not intended to be unambiguous or disconnected from the economic, financial and biotechnological conditions of CUK, DRC. It is therefore well established that the DRC and other poor African countries do not allocate a substantial budget to health structures having to organize and secure blood transfusion [4] [15] [16].

Using Mindraychemiluminescence as the gold standard, HCV seroprevalence was estimated to be around $29.5 \%$ in the present study. However, the seroprevalences found by the different TRODs showed a certain variability of the results around $27 \%$ and $32 \%$, with the highest prevalence for the Encode test, the result of which is well reflected by the likelihood and concordance measures found in the present study.

Compared to the study by Sumbu, et al. (8/4), the prevalence of anti HCV antibodies by the Mindray test in the present study was 15 times higher. The size of the sample, the demographic characteristics and probably the diagnostic method used can explain this difference. Indeed, the Mindray test uses the principle of chemiluminescence assay which has demonstrated a good diagnostic performance in the assay of hormones but especially in that of hepatic markers (HCV, HBV) with regard to previously popular methods such as RIA and conventional ELISA [17]. CLIA is currently widely used for screening for anti-HCV antibodies. This method is known to have excellent reliability, good precision, technical simplicity and being completely automated and less demanding of highly qualified technical expertise [18] [19]. Previous studies have shown improved specificity, high positive predictive value, and similar sensitivity compared to conventional ELISA for detection of anti-HCV antibodies. In addition, CLIA has the advantage of having a short, very fast turn-over and short turn-around time, which is a great advantage especially in high volume hospital laboratories [20]. The intrinsic and extrinsic characteristics of the various TRODs in the present study were found to be very good (Accurate, SD Bioline, Fortress) and quite good (Encode) compared to the Mindray benchmark test. The problem lies in the fact of their official acceptance as a diagnostic orientation test and screening for HCV. Indeed, WHO recently developed hepatitis C testing guidelines that are intended to serve as a basis for developing national guidelines for hepatitis testing in resource-limited settings [21]. The formulation of the recommendations was based on the GRADE (Grading of Recommendations, Assessment, Development and Evaluation) approach which includes an assessment of the quality of the evidence, but also considerations on the acceptability and preferences of patients and health workers., resource use and feasibility [21] [22].

In a survey of 22 programs on hepatitis in general and hepatitis $C$ in particular, 19 of which concerned countries with low and intermediate resources where access to laboratory infrastructure and specialized tests is limited, including DRC, $2 / 3$ of these programs use RDTs alone for screening and diagnostic referral for $\mathrm{HCV}$ infection. In the DRC, the programs concerned (non-governmental organization/NGO, International Organization/IO) did not include enzyme-linked 
immunosorbent assays or NAT tests [23].

No other study in the literature has worked on the TRODs assessed in this study. However, the study by Stéphane Chevaliez, et al. on new virological screening tools for hepatitis $\mathrm{C}$ and B reported a sensitivity, specificity, $\mathrm{L}+$ and $\mathrm{L}$ - respectively of $93.5 \%, 99.5 \%, 186.4$ and 0.038 for the SD Bioline test. These diagnostic performance indicators are better than those found in the present study [24]. Another study conducted in Cameroon evaluated 3 RDTs including First ResponseR, MultisureR and ToyoR. With the exception of the Toyo test for strict regulatory approval, the other tests were CE marked, i.e. European conformity [11]. In the same study, the sensitivities and specificities of First Response, Multisure and Toyo were 96\%/90\%, respectively; 96\%/78\% and $99 \% / 83 \%$. However, no test evaluated in this study met European Union standards. Indeed, according to European Union standards, anti-HCV tests had to have a sensitivity and specificity of $100 \%$ and $>99.5 \%$, respectively, to be approved for marketing. In addition, European standards are based on various studies carried out in Europe where the conditions for carrying out analyzes are practically different from those in countries with limited resources in terms of compliance with quality assurance [11].

Several facts must be mentioned in view of the performance of the tests evaluated in this study: the intrinsic quality of the reagents; the storage conditions, technicality and acceptability conditions of TROD in diagnostic strategies or even HIV co-infection [25]. In addition, no evaluated test was either CE marked, had received FDA approval, or had not yet been prequalified by the WHO. It is important to note that the majority of RDTs perform better in studies conducted in industrialized countries than in developing regions [26]. The WHO, has demonstrated the high probability of obtaining false results with TRODs if quality assurance is not respected [25].

\subsection{Implications of the Study}

The results of the present study will enable the Congolese government, via the Ministry of Health, to invest in hepatitis screening through programs that involve targeted tests on high-risk populations such as injecting drug users (IDU)., men who have sex with men (MSM), sex workers and prisoners, but also pregnant women and health workers. Unfortunately, these programs are supported by non-governmental organizations and international organizations (NGOs/IOs) than by the governments of the countries concerned, especially in sub-Saharan Africa (SSA), including the DRC [23]. The purpose of screening for infection would be to determine HCV viraemia; That is, in an anti-HCV antibody test, an additional test for HCV-core antigen (HCV-core Ag) or HCV RNA will be required (107/27). TRODs and laboratory immunoassays should meet quality and performance standards for sensitivity and specificity. TRODs will be an acceptable alternative when laboratory services are not available [24] [27] [28]. Ideally, countries should define screening strategies that take into account the local epi- 
demiology (prevalence, high risk group for HCV infection) and this within the framework of national plans [24]. Unfortunately, national plans are not developed in countries with limited resources. As part of the HCV TRODs, the majority of testing goes unchecked, as countries do not have strict supply chain regulatory policies.

The results generated by this study will facilitate the annual estimation of screening and access to routine screening as a complementary tool suitable for voluntary blood donors. Thus, the TRODs will be available to a large number of biological matrices including serum, plasma, venous blood, saliva, clavicular fluid (fluid secreted by the anterior groove of the gum and lip) as well as capillary blood.

The TRODs evaluated in this study will be essential components of the strategy for the scientific management (etiology, epidemiology, diagnosis, treatment and prevention) of HCV infection in voluntary blood donors. From now on, the CUK Clinical Biology Department will adopt a rigorous intellectual approach in interpreting the results produced by the TRODs which will lead to the medical decision: confirm or deny the presence of HCV infection; prescribe anti-HCV pharmacological molecules and ensure transfusion safety.

\subsection{Strengths and Limitations of the Study}

The present study has shown some limitations. Indeed, the lack of financial means made that it considered only 4 TRODs out of the multitude of TRODs circulating on the Congolese market. In addition, she used the enzyme-linked immunosorbent assay method which looks for anti-HCV antibodies instead of RT-PCR, which has the advantage of being even more precise by demonstrating HCV RNA. However, this study has the merit of having certain strengths. This is the first study in the DRC to assess the diagnostic performance of HCV TROD. It also gives the image and seriousness of the situation in relation to the HCV TRODs placed on the Congolese market.

\section{Conclusion}

The results of this study demonstrate the importance of evaluating diagnostic tests before final validation and authorization to market them. Indeed, the diagnostic performance of the TRODs evaluated in the present study was good according to the Mindray/chemiluminescence benchmark assay, but no TROD met European Union standards. Likewise, not all TRODs prefigured the WHO prequalification list or that of other international organizations. In addition, these performances are pejorative not only in the context of transfusion safety but also for patients, some of whom do not have the possibility of accessing the HCV PCR tests for the confirmation of the diagnosis, the treatment and the prognosis of the viral hepatitis $\mathrm{C}$. Thus, the Congolese government through the Ministry of Health/Laboratory Directorate should initiate large-scale studies for the validation of TRODs in circulation on the Congolese market taking into ac- 
count WHO guidelines.

\section{Authors' Contribution}

All authors that contributed to data analysis, drafting or revising the article, have agreed on the journal to which the article will be submitted, gave final approval of the version to be published, and agree to be accountable for all aspects of the work.

\section{Conflicts of Interest}

The authors declare no conflicts of interest for this work.

\section{References}

[1] OMS (2019) Hépatite C diagnostic, prise en charge et world gastroenterology organisation global guideline anal.

[2] Ministère de la SANTE RDC, Rapport PNTS 2017.

[3] Muwonga, J., Mulumba, J.A., Vercruysse, V., Kayembe, N., Gerad, C., Bwassa, J.P., Sonda, G.D. and Margraff, V. (2000) Séroprévalence du virus de l'hépatite C chez les donneurs de sang à Kinshasa. Vox sanguinis, 402.

[4] Sumbu, B.M.M., Longo-Mbenza, B., Nganga-Nkanga, M., Masidi, J.M., Nzongola-Nkasu, D.K., Ferdinand, M.K., Apalata, T. and Ahuka-Mundeke, S. (2016) Séroprévalence du Virus de l'Hépatite C chez les Donneurs de Sang aux Cliniques Universitaires de Kinshasa: 2005-2006 \& 2008-2013. Journal of Innovation and Research in Health Sciences and Biotechnology, 1, 140-149.

[5] Pioche, C., Léon, L., Larsen, C., et al. (2015) Dépistage Des Hépatites B et C en France en 2013, ENQUÊTE LABOHEP, Institut de veille sanitaire, Saint-Maurice, France BEH 26-2728.

[6] Dufour, D.R., Talastas, M., Fernandez, M. and Harris, B. (2003) Chemiluminescence Assay Improves Specificity of Hepatitis C Antibody Detection. Clinical Chemistry, 49, 940-944. https://doi.org/10.1373/49.6.940

[7] Pivert, A. and Lunel, F. (2006) Intérêt de la charge virale au cours de l'hépatite C. Antibiotiques, 8, 79-84. https://doi.org/10.1016/S1294-5501(06)70801-9

[8] Neuza, S.S. (2011) Laboratorial Diagnosis of Syphilis. In: Neuza, S.S., Ed., Syphilis-Recognition, Description and Diagnosis, InTech Inc., Croatia, 87-108.

[9] Seña, A.C., White, B.L. and Sparling, P.F. (2010) Novel Treponema Pallidum Serologic Tests: A Paradigm Shift in Syphilis Screening for the 21st Century. Clinical Infectious Diseases, 51, 700-708. https://doi.org/10.1086/655832

[10] Madiya, M., Sagar, S., Vishwanath, S., Banerjee, B., Eshwara, V.K. and Chawla, K. (2016) Comparing Assay Performance of ELISA and Chemiluminescence Immunoassay in Detecting Antibodies to Hepatitis B Surface Antigen. Journal of Clinical and Diagnostic Research, 10, DC22-DC25. https://doi.org/10.7860/JCDR/2016/24108.8921

[11] Fondjo, C.L.K., Ngoupo, P.A.T., Ngono, L., Plantier, J.-C. and Njouom, R. (2018) Performance Evaluation of Three Rapid Screening Assays for Detection of Antibodies to Hepatitis C Virus in Cameroun. BMC Research Notes, 11, 352. https://doi.org/10.1186/s13104-018-3465-8

[12] Gao, F., Talbot, E.A., Loring, C.H., Power, J.J., Dionne-Odom, J., Alroy-Preis, S., et 
al. (2014) Evaluation of Performance of the OraQuick ${ }^{(\mathrm{R})}$ HCV Rapid Antibody Test for Screening Exposed Patients in a Hepatitis C Outbreak Investigation. Journal of Clinical Microbiology, 52, 2650-2652. https://doi.org/10.1128/JCM.00132-14

[13] Ruopp, M.O., Comb, B.W.W. and Schisterman, E.F. (2008) Youden Index and Optimal Cut-Point Estimated from Observations Affected by a Lower Limit of Detection. Biometrical Journal, 50, 414-430. https://doi.org/10.1002/bimj.200710415

[14] Loua, A., Magassouba, F.B., Camara, M., Haba, N.G. and Balde, A.M. (2004) Bilan de 4 ans de sérologie VIH au centre national de transfusion sanguine de Conakry. Bulletin de la Société de Pathologie Exotique, 97, 139-141.

[15] Mayaki, Z., Dardenne, N., Kabo, R., Moutschen, M., Sondag, D., Albert, A. and Gérard, C. (2013) Séroprévalence des marqueurs de l'infection chez les donneurs de sang à Niamey (Niger). Elsevier Masson. https://doi.org/10.1016/j.tracli.2013.03.053

[16] Te, H.S. and Junsen, D.M. (2010) Epidemiology of Hepatitis B and C Viruses: A Global Overview. Clinics in Liver Disease, 14, 1-21.

https://doi.org/10.1016/j.cld.2009.11.009

[17] Dudley, R.F. (1990) Chemiluminescence Immunoassay: An Alternative to RIA. Laboratory Medicine, 21, 216-222. https://doi.org/10.1093/labmed/21.4.216

[18] AvnerHershlag, M.Z., Lesser, M. and Scholl, G. (2000) Is Chemiluminescent Immunoassay an Appropriate Substitution for Radioimmunoassay in Monitoring Estradiol Levels? Fertility and Sterility, 73, 1174-1178.

https://doi.org/10.1016/S0015-0282(00)00514-8

[19] Huh, H., Chae, S.-L. and Cha, Y.J. (2007) Comparison Study with Enzyme Immunoassay and Chemiluminescence Immunoassay for Hepatitis B Virus Surface Antigen Detection. The Korean Journal of Laboratory Medicine, 27, 355-359. https://doi.org/10.3343/kjlm.2007.27.5.355

[20] World Health Organization (2017) Guidelines on Hepatitis B and C Testing. Geneva. http://apps.who.int/iris/bitstream/10665/254621/1/9789241549981

[21] Guyatt, G., Oxman, A.D., Akl, E.A., Kunz, R., Vist, G., Brozek, J., et al. (2011) GRADE Guidelines: 1. Introduction-GRADE Evidence Profiles and Summary of Findings Tables. Journal of Clinical Epidemiology, 64, 383-394. https://doi.org/10.1016/i.jclinepi.2010.04.026

[22] World Health Organization (2014) Handbook for Guideline Development. Geneva. http://apps.who.int/medicinedocs/documents/s22083en/s22083en.pdf

[23] Ishizaki, A., Bouscaillou, J., Luhmann, N., Liu, S., Chua, R., Walsh, N., Hess, S., Ivanova, E., Roberts, T. and Easterbrook, P. (2017) Survey of Programmatic Experiences and Challenges in Delivery of Hepatitis B and C Testing in Low- and Middle-Income Countries. BMC Infectious Diseases, 17, Article No. 696. https://doi.org/10.1186/s12879-017-2767-0

[24] Chevaliez, S. and Pawlotsky, J.M. (2018) New Virological Tools for Screening, Diagnosis and Monitoring of Hepatitis B and C in Resource-Limited Settings. Journal of Hepatology, 69, 916-926. https://doi.org/10.1016/j.jhep.2018.05.017

[25] Jewett, A., Smith, B.D., Garfein, R.S., Cuevas-Mota, J., Teshale, E.H. and Weinbaum, C.M. (2012) Field-Based Performance of Three Pre-Market Rapid Hepatitis C Virus Antibody Assays in STAHR (Study to Assess Hepatitis C Risk) among Young Adults Who Inject Drugs in San Diego, CA. Journal of Clinical Virology, 54, 213-217. https://doi.org/10.1016/j.jcv.2012.04.003

[26] Adler, M., Behel, S., Duncan, D., Houston, J.M., Kalou, M., Lasry, A., et al. (2015) Consolidaded Guidelines on HIV Testing Services: 5Cs: Content, Confidentiality, 
Counselling, Correct Results and Connection. World Health Organization, Geneva.

[27] Khuroo, M.S., Khuroo, N.S. and Khuroo, M.S. (2015) Diagnostic Accuracy of Point-of-Care Tests for Hepatitis C Virus Infection: A Systematic Review and Meta-Analysis. PLoS ONE, 10, e0121450. https://doi.org/10.1371/journal.pone.0121450

[28] Guide de validation des méthodes d'analyses. Anses-Pôle Recherche et Référence-ANSES/PR3/07/01 octobre 2015. 1-67. 